\title{
Wet peroxide oxidation of phenol over mixed pillared montmorillonites
}

\author{
Manju Kurian, S. Sugunan* \\ Department of Applied Chemistry, Cochin University of Science and Technology. Kochi 682022. India \\ Received 6 November 2004: received in revised form 26 February 2005; accepted 25 August 2005
}

\begin{abstract}
Wet peroxide oxidation (WPO) of phenol is an effective means for the production of diphenols, which are of great industrial importance. An added advantage of this method is the removal of phenol from wastewater effluents. Hydroxylation of phenol occurs efficiently over mixed iron aluminium pillared montmorillonites. An initial induction period is noticed in all cases. A thorough study on the reaction variables suggests free radical mechanism for the reaction.
\end{abstract}

(c) 2005 Elsevier B.V. All rights reserved.

Keywords: Pillared clays; Phenol hydroxylation; Brönsted acidity; Free radicals

\section{Introduction}

Phenol is a major water pollutant. The presence of phenol even in trace amounts has been proven fatal to living beings. Among catalysed wet oxidations, oxidation with hydrogen peroxide is preferred because the active oxygen content of hydrogen peroxide is much higher than other oxidants. Wet peroxide oxidation (WPO) with hydrogen peroxide can bring about removal of phenol from effluents. Water is the only bye product formed and the oxidant is inexpensive. Also, aqueous hydrogen peroxide is a stable reagent, provided it is handled and stored in the proper manner [1]. An added advantage of the reaction is that oxidation of phenol produces catechol and hydroquinone, two important intermediates in agrochemical and fine chemical industries. Catechol and hydroquinone are also used as photographic developers and antioxidants. Conventionally, $\mathrm{HClO}_{4}$ $\mathrm{H}_{3} \mathrm{PO}_{4}$ and $\mathrm{Fe}$ (III)/Co(II) catalysts were used for oxidation of phenol [2,3]. But obvious shortcomings of these homogeneous catalysts prevent their wide use in diphenol production. Titanium silicalite-1 (TS-I), a titanium containing zeolite was found superior to other catalysts for phenol hydroxylation, duc to decreased formation of tar and polluting byc products and subsequently the method was commercialised. Extensive study of the reaction has been conducted using this catalyst [4.5]. Though TS-I is

\footnotetext{
- Corresponding author. Tel.: +91 4842575804.

E-mail address: ssg@cusat.ac.in (S. Sugunan).
}

an excellent catalyst for phenol hydroxylation, lack of thermal stability and somewhat complex preparation method have led researchers to develop other cheaper catalysts for the hydroxylation of phenol. Since peroxide is used, free radical mechanism can be expected for the reaction and hence various iron and copper containing catalysis have been employed [6-9].

Pillar interlayered clays constitute a novel class of microporous materials with good thermal stability, high surface area and pronounced Brönsted and Lewis acidity. These matcrials result from a two step modification of naturally occurring swelling clay minerals; the propping apart of clay layers by intercalation with oligo or polymeric cationic metal complexes and calcination of the intercalated clays transforming the inserted complexes into nanosized pillars of metal oxides cross linked to the clay layers. Several processes of industrial importance have been reported over pillared clays [10-13]. Here we report the use of iron aluminium mixed pillared montmorillonite and its transition metal exchanged analogues for the hydroxylation of phenol.

\section{Experimental}

The mixed pillared clay was synthesised by partial hydrolysis of $0.1 \mathrm{M} \mathrm{Fe}\left(\mathrm{NO}_{3}\right)_{3}$ and $\mathrm{Al}\left(\mathrm{NO}_{3}\right)_{3}$ solution (equimolar ratio) by drop wise addition of $0.3 \mathrm{M} \mathrm{Na} \mathrm{CO}_{3}$ solution under vigorous stirring. Intercalation of pillaring species into the clay layers was done by treating the pillaring solution with a previously swollen clay suspension at $80^{\circ} \mathrm{C}[\mathrm{OH} /$ metal ratio, 2 
and metal/clay ratio, $20 \mathrm{mmol} / \mathrm{g}$ clay]. The clay after exchange was washed several times with distilled water and filtercd. This was dried in air oven at $110^{\circ} \mathrm{C}$ overnight, followed by calcination for $6 \mathrm{~h}$ at $450^{\circ} \mathrm{C}$ in muffle furnace. Exchange with transition metals was donc using 0.1 molar aqueous solutions of the corresponding metal nitrate. The clay after exchange was washed five to six times with distilled water. This was filtered and dried in air oven at $110^{\circ} \mathrm{C}$ overnight and calcined for $5 \mathrm{~h}$ at $500 \mathrm{C}$. The pillared clays synthesised for the present study are notated as $\mathrm{X} / \mathrm{FeAl} \mathrm{PM}$, where $\mathrm{X}$ is the transition metal exchanged. The diffractometer traces of the catalyst samples were taken in RIGAKU D/MAX-C instrument using $\mathrm{Cu} \mathrm{K} \alpha$ radiation $(\lambda=1.5405 \AA)$. The simultaneous determination of surlace areas and pore volumes of the catalyst samples was done on a Micromeritics Gemini analyser. NMR spectra of samples were recorded by a $300 \mathrm{DSX}$ Brucker spectrometer. Cumene cracking was carried out in vapour phase at atmospheric pressurc. The products were analysed using Chemito 8610 Gas Chromatograph equipped with fiame ionisation detector and FFAP column.

The liquid phase hydroxylation of phenol was carried out in a $50 \mathrm{~mL}$ round bottomed flask equipped with air condenser and magnetic stirrer. $0.1 \mathrm{~g}$ of the catalyst, preactivated at $500^{\circ} \mathrm{C}$ for $2 \mathrm{~h}$ was added to calculated amounts of phenol and solvent. Required amounts of $30 \% \mathrm{H}_{2} \mathrm{O}_{2}$ was added drop wise to the reaction medium and reaction ensued at specified temperatures. Reaction mixture was periodically analysed using gas chromatography. Benzoquinone was not detected in any case. Tarry products were obscrved in all runs. Quantitative cstimation of tarry products was not pursued and conversion of phenol refers to conversion to diphenols. Thus, percentage conversion (wt.\%) of phenol is the total percentage of phenol transformed into diphenols.

\section{Results and discussion}

\section{I. Surface area and pore volume measurements}

The surface area and pore volume of the prepared systems are given in Table 1. Montmorillonite has a BET surface arca of $14.3 \mathrm{~m}^{2} \mathrm{~g}^{-1}$ and Langmuir surface arca of $27.9 \mathrm{~m}^{2} \mathrm{~g}^{-1}$. As a result of pillaring, surface area and pore volume increases dras-

Table 1

Surface area and pore volume of the systems

\begin{tabular}{lccl}
\hline Catalyst & \multicolumn{2}{l}{ Surface area $\left(\mathrm{m}^{2} \mathrm{~g}^{-1}\right)$} & Pore volume \\
\cline { 2 - 3 } & BET & Langmuir & \\
\hline V/FeAl PM & 158.3 & 229.9 & 0.1753 \\
Mn/FeAl PM & 156.0 & 219.2 & 0.1703 \\
Co/FeAl PM & 164.9 & 229.5 & 0.1871 \\
Ni/FeAl PM & 142.2 & 203.9 & 0.1673 \\
Cu/FeAl PM & 144.5 & 207.6 & 0.1658 \\
Zn/FiAl PM & 147.2 & 233.6 & 0.1635 \\
FeAl PM & 170.6 & 253.7 & 0.1894 \\
$M^{h}$ & 14.3 & 27.9 & 0.0058 \\
\hline
\end{tabular}

"Pore volume measured at $0.9976 \mathrm{P} / \mathrm{P}_{\text {() }}$.

h Montmorilkonite KSF. tically. Transition metal exchange decreases the surface area and pore volume, mainly in external surlace. Pillaring is the process by which stable metal oxide clusters are incorporated into interlayer space of swellable clays. As a result, a three dimensional porous network is created. Hence surface area and pore volume increases cxtensively, especially the microporous surface area. The external surface area in pillared clays arises from mesopores, which are mainly interparticle voids. Transition metal exchange deposits the metal oxides inside the porous network and hence the decrease in surface area (especially the external surface) and pore volume.

\subsection{X-ray diffraction}

With the exception of surface area and pore volume measurements, the easiest way to determine whether pillar intercalation is successful is to record the X-ray diflraction pattern of an oriented film of the product. Fig. I depicts XRD patterns of montmorillonite and FeAl PM. Pillared clays are semi crystalline in nature. The broad bands obtained in the XRD spectrum, instead of sharp peaks can be attributed to semi crystalline nature of clays. Hence indexing of the spectrum is not possible for this type of solid acids. The only data that can be obtained is the $d$ spacing of $(001)$ plane, which indicates the extent of propping apart of clay layers. The characteristic $d_{001}$ spacing of montmorillonite increased from $9.8 \AA$ to $17.8 \AA$ for FeAl PM. Shifting of $2 \theta$ values clearly suggests expansion of clay layer during pillaring process. The increase in $d$ spacing of $(001)$ plane to $17.8 \AA$ is indicative of the presence of $\mathrm{Al}_{13}$ like polymers. This polymer with structural formula, $\left[\mathrm{AlO}_{4} \mathrm{Al}_{12}(\mathrm{OH})_{24}\left(\mathrm{H}_{2} \mathrm{O}\right)_{12}\right]^{7+}$ is a tri-decamer composed of one aluminium tetrahedra surrounded by 12 aluminium octahedra. It contains four layers of superimposed oxygen atoms needed for expanding clay basal spacings to $18 \AA$. Formation of mixed $\mathrm{Al}_{13-x} \mathrm{Fe}_{x}$ pillars, based on the $\mathrm{Fe}$ content of the pillared solids was reported by Bergaya et al. [14]. The effect of exchange with transition metals on the XRD patterns was studied for representative samples. The XRD patterns were exactly identical to that of montmorillonite. Thus, it can be concluded that insertion of the second metal after the formation of stable pillars does not destabilise the porous network. Additional peaks corresponding to the exchanged metal oxides were

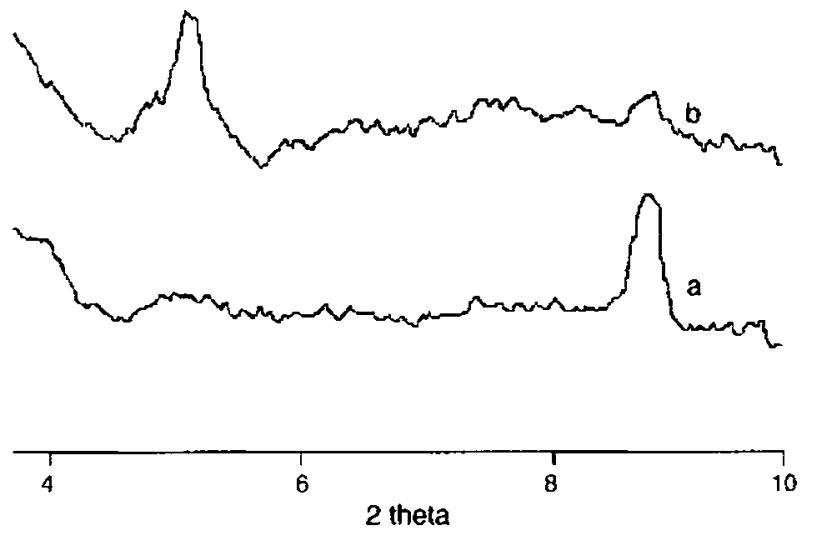

Fig. 1. XRD profile: (a) M: (b) FeAl PM. 


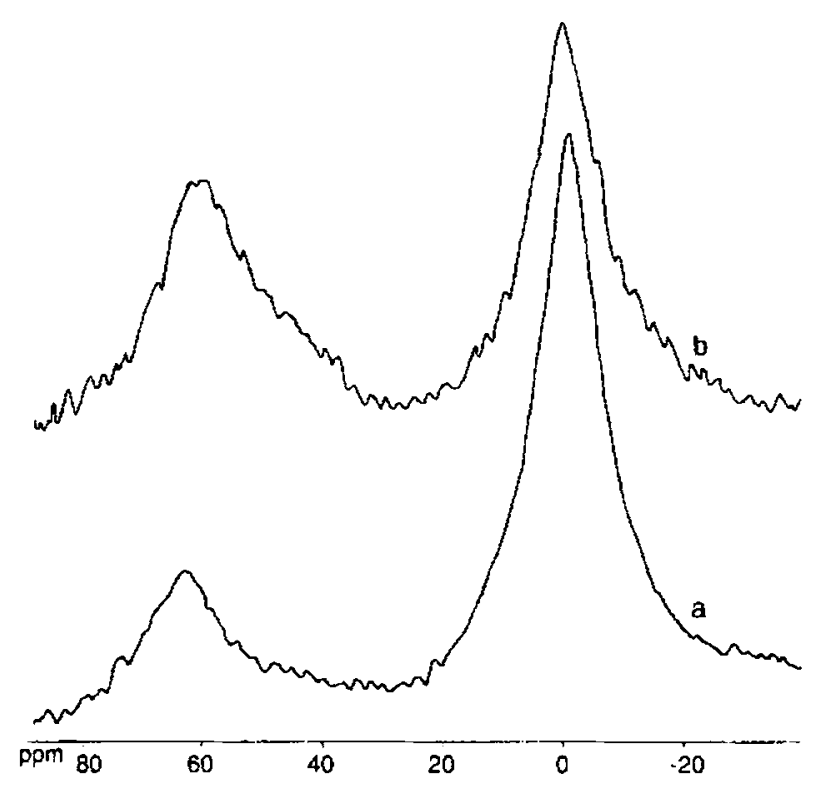

Fig. 2. ${ }^{27}$ Al NMR spectra: (a) M; (b) FeAl PM.

not noticed. This may be due to the diminutive amounts of the exchanged metal in these samples.

\section{3. ${ }^{27}$ Al NMR spectroscopy}

Clay minerals and pillared clays have been the subject of several solid state NMR investigations [15]. The ${ }^{27}$ Al NMR spectra of the pillared clays are illustrated in Fig. 2. Montmorillonite shows two resonances; one at $+1.38 \mathrm{ppm}$ that can be ascribed to octahedral $\mathrm{Al}$ atoms and other at $+66.0 \mathrm{ppm}$ attributable to tetrahedrally coordinated $\mathrm{Al}$ atoms. The ${ }^{27} \mathrm{Al}$ NMR spectrum for FeAl PM shows the $\mathrm{Al}^{\mathrm{IV}}$ signature of Keggin cation at $+65.8 \mathrm{ppm}$. Resonance corresponding to octahedral Al aroms also is shifted to $+1.97 \mathrm{ppm}$. Thus the pillaring solution would be made from these cations in which $\mathrm{Al}^{3+}$ is partially replaced by $\mathrm{Fe}^{3+}$. The nature of substitution is not known, but in view of Al by Fe in octahedral layers of micas, it is likely that some of the octahedral $\mathrm{Al}$ atoms in Keggin structure are replaced by Fe atoms [16]. Thus, structure of iron aluminium mixed pillared systems is similar to Al polymeric species and not hydroxy iron oligomers. Also, relaxation effects caused by iron paramagnetic centres are operative. Hence full width at half maximum (FWHM) of the peaks is greater, compared to montmorillonite. The effect of transition metal exchange on structural stability of pillared clay was examined by taking ${ }^{27} \mathrm{Al}$ NMR spectra of copper and cobalt doped systems as representatives. As a result of exchange with transition metals, peak width as well as peak positions do not vary. This shows that incorporation of transition metals does not affect the structural stability of layers and pillars.

\subsection{Cumene cracking}

Cumene is a conventional model compound for testing the catalytic activity since it undergoes diverse reactions over different types of acid sites. Major reactions taking place during cracking of cumene are dealkylation or cracking to benzenc
Table 2

Catalytic activity of various systems towards cumene cracking

\begin{tabular}{lllll}
\hline Catalyst & Conversion $(\%)$ & \multicolumn{2}{l}{ Selectivity (\%) } \\
\cline { 3 - 4 } & & $\begin{array}{l}\text { a-Methyl } \\
\text { styrene }\end{array}$ & $\begin{array}{l}\text { Dealkylation } \\
\text { products }^{a}\end{array}$ & $\begin{array}{l}\text { Lewis/ } \\
\text { Brönsted }^{b}\end{array}$ \\
\hline V/FeAl PM & 22.8 & 54.3 & 45.7 & 1.19 \\
Mn/FcAl PM & 22.5 & 53.2 & 46.8 & 1.14 \\
Co/FeAl PM & 32.5 & 54.2 & 45.8 & 1.18 \\
Ni/FeAl PM & 28.9 & 60.7 & 39.3 & 1.54 \\
Cu/FeAl PM & 19.5 & 50.2 & 49.8 & 1.0 \\
Zn/FeAl PM & 21.1 & 53.3 & 46.7 & 1.14 \\
FeAl PM & 20.7 & 62.7 & 36.3 & 1.73 \\
\hline
\end{tabular}

Temperature, $400^{\circ} \mathrm{C}$; WHSV, $7 \mathrm{~h}^{-1}$; time on stream. $2 \mathrm{~h}$.

"Benzene, toluene, eihyl benzene.

h Ratio of $\alpha$-methyl styrenc and dealkylated products selectivity.

and propene and dehydrogenation to $\alpha$-methyl styrene. Small amounts of ethylbenzene and toluene can be formed by cracking of side chain, which on dehydrogenation gives styrene. Cracking of cumene is generally attributed to Brönsted acid sites by a carbonium ion mechanism whereas $\alpha$-methyl styrene is formed on Lewis acid sites [17].

The catalytic performance of prepared materials for the reaction and the effect of transition metal exchange under optimised conditions are given in Table 2 . Ethylbenzene and styrene appeared in minor quantities and in some cases toluene also was detccted. All the dealkylated products are bannered together and Lewis to Brönsted acid ratio gives the ratio between the dehydrogenated and cracked products. FeAl PM shows cumene conversion of $20.7 \%$ with a selectivity of $62.7 \%$ for $\alpha$-methyl styrenc. Transition metal exchange decreases the dehydrogenation activity with a concomitant decrease in catalytic activity. The high selectivity for the dehydrogenated product can be attributed to the effective pillaring. It has been well documented that the activity for cumene cracking reaction can be correlated to the total acidity of the catalyst. Also, dehydrogenation to $\alpha$-methyl styrene occurs over Lewis acid sites and cracking over Brönsted acid sites. The decrease in activity for cobalt and zinc exchanged systems can be traced to decrease in $\alpha$ methyl styrene selcctivity. Hence decrease in the number of acid sites is mainly due to decrease in the number of Lewis acid sites. NMR spectroscopy cvidences the exchanged metal to be present inside the porous network thus reducing the accessibility to Lewis acid sites, which are resident mainly in the pillars.

\subsection{Phenol hydroxylation}

Table 3 discloses the catalytic performances of various mixed pillared systems towards hydroxylation of phenol with hydrogen peroxide. FeAl PM exhibits very high activity for the reaction. Almost $80 \%$ of the phenol gets converted over this catalyst within $45 \mathrm{~min}$, with $38 \%$ selectivity towards hydroquinone. Incorporalion of transition metals into the porous network reduces the activity of pillared clay especially for copper and zinc exchanged systems. The product selectivity however remains almost the same. 
Table 3

Activity of iron aluminium pillared systems towards phenol hydroxylation

\begin{tabular}{llll}
\hline Catalyst & Conversion (\%) & \multicolumn{2}{l}{ Selectivity (\%) } \\
\cline { 3 - 4 } & & Catechol & Hydroquinone \\
\hline V/FeAl PM & 77.2 & 64.1 & 3.9 \\
Mn/FeAl PM & 72.3 & 63.4 & 36.6 \\
Co/PeAl PM & 77.1 & 66.0 & 34.0 \\
Ni/FeAl PM & 71.1 & 63.8 & 36.2 \\
Cu/FeAl PM & 58.2 & 67.7 & 32.3 \\
Zn/FeAl PM & 58.9 & 63.5 & 36.5 \\
FeAl PM & 79.0 & 62.0 & 38.0 \\
\hline
\end{tabular}

Phenolsolvent: $\mathrm{H}_{2} \mathrm{O}_{2}$ (molar ratio), $1: 5: 5$; catalyst, $0.1 \mathrm{~g}$ : temperature, $80 \cdot \mathrm{C}$ : time, $45 \mathrm{~min}$

The high activity of iron containing systems can be attributed to the redox nature of iron. Since hydrogen peroxide is used, there is possibility for involvement of free radicals and presence of iron in the catalyst can produce free radicals. Catechol has been postulated to be formed in the external surface of the catalyst where as hydroquinone is formed inside the pores $[18,19]$. The diffusion hindrance of reactants into the porous network of the pillared clay can be the reason for reduced hydroquinone selectivity, though thermodynamically 0 - and $p$-products have cqual probability.

Though acidity is not an important parameter for oxidation reactions, several reports are present in literature suggesting the participation of Brönsted acid sites in hydroxylation of phenol $[20,21]$. The availability of structural $\mathrm{OH}$ species (Brönsted acidity) in zeolites has been correlated with catalytic efficiency. In clays, Brönsted acidity stems mainly from structural $\mathrm{OH}$ groups. Hence an atcmpt is made to correlate observed activities with Brönsted acidity as obtained from cumene cracking reaction (Fig. 3). Brönsted acidity ties well with catalytic activity. An inconsistency is observed for FeAl PM that can be explained on the basis of increased hydroquinone formation inside pores. Thus, it can be conclusively noted that Brönsted acidity play prominent role in phenol hydroxylation.

The question about the true nature of the reaction is a popular subject of concern for the scientific community. Leaching

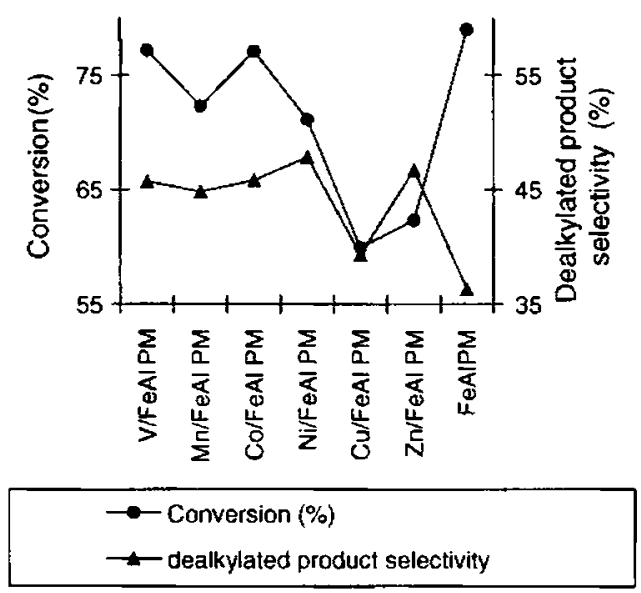

Fig. 3. Dependence of catalytic activity un Brönsted acidity (as obtained from cumene cracking reaction)
Table 4

Effect of metal leaching

\begin{tabular}{llll}
\hline Catalyst & Conversion (\%) & \multicolumn{2}{l}{ Sclectivity (\%) } \\
\cline { 3 - 4 } & & Catechol & Hydroquinone \\
\hline 45 & 77.2 & 64.1 & 35.9 \\
$25^{4}$ & 78.5 & 6.3 .5 & 36.5 \\
\hline
\end{tabular}

Phenol:solvent: $\mathrm{H}_{2} \mathrm{O}_{2}$ (molar ratio). 1:5:5: catalyst. $0.1 \mathrm{~g}$; temperature. $80 \mathrm{C}$.

i After filtering off the catalyst.

of metals from the catalyst surface can occur without much transformation in reaction profile, gradually changing the nature of reaction from truly helerogeneous to partly homogeneous. Leaching of certain metals like $\mathrm{Fe}$ in alkylation reactions and $\mathrm{V}$ in oxidation reactions has been reported $[22,23]$. This can lead to structural collapse as well as partly homogeneous reaction. Hence the effect of leaching of the active phase was studied at $35 \mathrm{~min}$, by continuing the reaction for further $25 \mathrm{~min}$, after filtering off the catalyst. The results are given in Table 4 . The percentage conversion and selectivity pattern show only a nominal variation (within the limits of GC error) even after 25 min. The diminutive increase in conversion\% signifies heterogeneity of the reaction. Thus it is confirmed that the reaction occurs exclusively on clay catalyst.

Hydroxylation of phenol is extremely sensitive to reaction conditions as well as quality of the catalyst. Hence the influence of various reaction variables like time, temperature, phenol concentration, oxidant concentration, solvent, solvent concentration and catalyst concentration was extensively studied using $\mathrm{V} / \mathrm{FeAl}$ PM as reference catalyst.

\subsection{Effect of time}

The effect of time on wet peroxide oxidation of phenol was studied at a range of $90 \mathrm{~min}$. The results are given in Fig. 4. From the figure it can be deduced that excellent conversion occurs even at $15 \mathrm{~min}$. However, an induction period of about $5 \mathrm{~min}$ was noticed, after which violent reaction starts, turning the reaction mixture black. This was observed at all temperatures.' The conversion\% does not alter much with increase in duration of run. After $45 \mathrm{~min}$, the activity of the catalyst drops. Regard- '

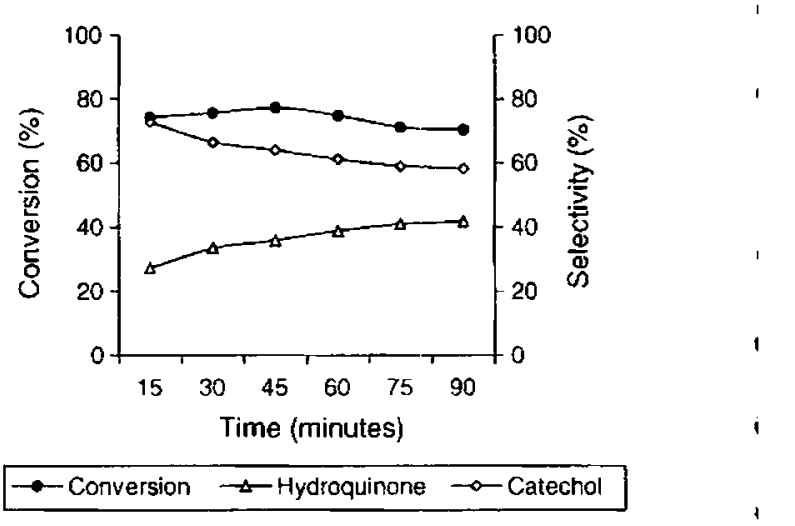

Fig. 4. Effect of time: phenol: $\mathrm{H}_{2} \mathrm{O}: \mathrm{H}_{2} \mathrm{O}_{2}$ (molar ratio). 1:5:5; catalyst, $0.1 \mathrm{~g}$; temperatuse, $80 \% \mathrm{C}$. 
ling selectivity pattern, hydroquinone selectivity improves with time.

The results indicate that, an optimum reaction time is needed for substantial phenol conversion. After a particular period, activity of surface sites practically drops. This change in activity pattern may be attributed to poisoning of surface sites by reaction products [24]. Tar, produced by over oxidation of diphenols is a major poison for surface sites. It has been reported that with zeolites and molecular sieve catalysts, catechol is formed in the external surface of catalyst, whereas hydroquinone is formed inside pores $[18,19]$. Since pillared clays also possess a porous network, shape selective nature can be assigned for this type of catalysts also. Hence formation of catechol is low in the pores. As time goes by, more and more reactants diffuse in and out of pores leading to increased hydroquinone selectivity.

\subsection{Effect of temperature}

The effect of temperature was checked in a range of $50-90^{\circ} \mathrm{C}$ at an interval of $10^{\circ} \mathrm{C}$ (Table 5). On an average, conversion of phenol increases with temperature. At room temperature, reaction does not occur even after $1 \mathrm{~h}$. As the temperature is increased by $10^{\circ} \mathrm{C}$, drastic change occurs in reaction rate. Maximum conversion is observed at $80^{\circ} \mathrm{C}$ and at $90^{\circ} \mathrm{C}$, conversion $\%$ drops. Regarding selectivity pattern, low temperatures favour

Table 5

Effect of time

\begin{tabular}{|c|c|c|c|c|}
\hline \multirow[t]{2}{*}{ Temperature ( $\mathrm{C}$ ) } & \multirow[t]{2}{*}{ Time (min) } & \multirow[t]{2}{*}{ Conversion $(\%)$} & \multicolumn{2}{|c|}{ Selectivity (\%) } \\
\hline & & & Catechol & Hydroquinone \\
\hline \multirow{5}{*}{50} & 30 & 17.4 & 28.8 & 71.2 \\
\hline & 45 & 27.5 & 40.3 & 59.7 \\
\hline & 60 & 29.6 & 46.3 & 53.7 \\
\hline & 75 & 31.8 & 64.7 & 35.3 \\
\hline & 90 & 29.9 & 51.3 & 48.7 \\
\hline \multirow{5}{*}{$1^{60}$} & 30 & 29.4 & 38.5 & 61.5 \\
\hline & 45 & 52.2 & 53.9 & 36.1 \\
\hline & 60 & 60.6 & 65.3 & 34.7 \\
\hline & 75 & 57.2 & 63.4 & 34.6 \\
\hline & 90 & 54.6 & 61.2 & 38.8 \\
\hline \multirow{5}{*}{170} & 30 & 64.3 & 69.6 & 30.4 \\
\hline & 45 & 66.1 & 67.0 & 33.0 \\
\hline & 60 & 61.3 & 58.2 & 41.8 \\
\hline & 75 & 69.4 & 65.1 & 34.9 \\
\hline & 90 & 71,0 & 68.1 & 31.9 \\
\hline \multirow{6}{*}{80} & 15 & 74.4 & 72.8 & 27.2 \\
\hline & 30 & 75.6 & 66.5 & 33.5 \\
\hline & 45 & 77.2 & 64.1 & 35.9 \\
\hline & 60 & 74.9 & 61.1 & 38.9 \\
\hline & 75 & 71.2 & 59.0 & 41.0 \\
\hline & 90 & 70.3 & 58.3 & 41.7 \\
\hline \multirow{5}{*}{$I_{90}$} & 15 & 62.7 & 54.2 & 45.8 \\
\hline & 30 & 67.6 & 61.4 & 38.6 \\
\hline & 45 & 69.9 & 62.8 & 37.2 \\
\hline & 60 & 70.8 & 62.7 & 37.3 \\
\hline & 75 & 72.9 & 58.8 & 41.2 \\
\hline 1 & 90 & 74.8 & 62.4 & 37.6 \\
\hline
\end{tabular}

, Phenol: $\mathrm{H}_{2} \mathrm{O}: \mathrm{H}_{2} \mathrm{O}_{2}$ (molar ratio). 1:5:5; catalysi, $0.1 \mathrm{~g}$. higk yields of hydroquinone. Another remarkable point is that at low temperatures, hydroquinone selectivity decreases with time. As the temperature is raised, hydroquinone selectivity suffers but increases with time at a particular temperature.

Induction period was noticed for the reaction at all temperalures, suggestive of free radical mechanism. The induction period shows inverse relationship with temperature. Hence the non-occurrence of the reaction at room temperature may be due to prolonged induction period. The sweeping enhancement in the amount of oxidised phenol with temperature, again is suggestive of free radical mechanism for the reaction. At higher temperatures, the formation of free radicals occurs to greater extent and hence the sudden boost in conversion\%. The decrease in catalylic activity at $90^{\circ} \mathrm{C}$ can be recognised with high rate of $\mathrm{H}_{2} \mathrm{O}_{2}$ decomposition. This side reaction suppresses the availability of sufficient peroxide for the reaction and is indicative of decreased activation energy for hydrogen peroxide decomposition compared to the oxidation reaction over clay catalyst [25].

\subsection{Effect of phenol concentration}

The influence of the amount of phenol in reaction mixture was studied by keeping the amount of water and hydrogen peroxide constant and altering the amount of phenol. The observations are presented in Fig. 5. From the figure, it can be noticed that a minimum phenol concentration is required for good conversions. The amount of phenol oxidised is directly dependant on the amount of phenol originally present in the reaction mixture. Nevertheless, after an optimum phenol level, conversion\% again drops. Commenting on selectivity pattern, one can easily notice that hydroquinone selectivity improves steadily with increase in the amount of phenol. Thus, at a phenol:water:hydrogen peroxide molar ratio of 4:5:5, almost an equal ratio of isomeric products is obtained.

At lower concentrations, due to solvent effects, phenol molecules may be experiencing difficulty in approaching active sites and hence the reduced activity. The decrease in phenol conversion at high concentrations can be attributed to the fact that conversions are calculated as weight $\%$ of unreacted phenol.

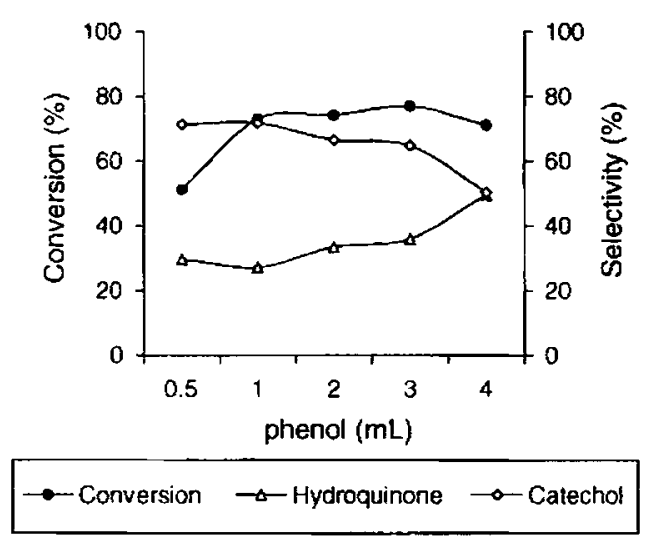

Fig. 5. Effect of phenol concentration. Water: $\mathrm{H}_{2} \mathrm{O}_{2}$ (molar ratio). 5:5; catalyst, $0.1 \mathrm{~g}$; temperature, $80^{\circ} \mathrm{C}$; time, $45 \mathrm{~min}$. 


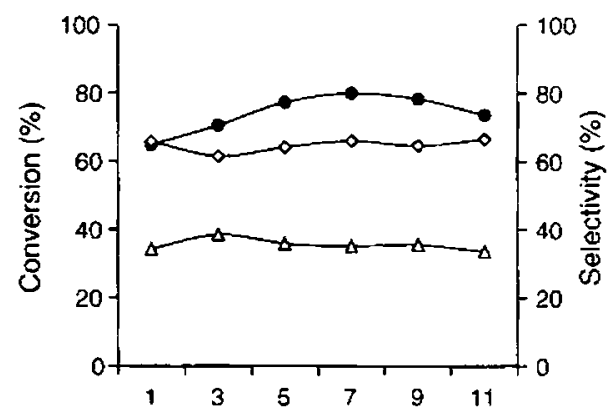

$$
\begin{aligned}
& \rightarrow \text { Conversion } \rightarrow-\text { Hydroquinone } \\
& \rightarrow \text { Caiechol }
\end{aligned}
$$

Fig. 6. Effect of oxidant concentration. Phenol:solvent (molar ratio), 1:5; catalyst, $0.1 \mathrm{~g}$ : temperature, $80^{\circ} \mathrm{C}$ : time, $45 \mathrm{~min}$.

Increase in phenol concentration increases the rate of diffusion of reactants into the pores and hence the increase in hydroquinone selectivity.

\subsection{Effect of peroxide concentration}

The amount of oxidant used is an essential parameter for oxidation reactions. The influence of phenol to hydrogen peroxide molar ratio is illustrated in Fig. 6. Oxidant present even in trace quantities can bring about oxidation of phenol to diphenols over pillared clay catalysts. Increase in amount of peroxide increases the activity. However, after an optimum amount of oxidant, the conversion\% falls. Hydroquinone selectivity also follows the same trend.

The proficient conversion of phenol over pillared clay catalysts depicts their superior ability for oxidation reactions. The presence of iron can be a reason for this. Maximum conversion of phenol to diphenols was observed at peroxide to phenol molar ratio of 7 . Several authors reported decrease in the reaction rate at high oxidant concentrations [26]. Neumann and Levin Elad proposed large excess of water accompanying hydrogen peroxide as possible reason for this [27]. This seems to be an unsatisfactory explanation since the catalytic activity was unaffected by solvent concentration (discussed in subsequent section). The increase in oxidant concentration increases the deep oxidation of produced diphenols to tar and this can be a possible explanation for decreased conversion. The high concentration of $\mathrm{H}_{2} \mathrm{O}_{2}$ can also accelerate self decomposition of the oxidant, reducing the conversion of phenol.

\subsection{Effect of solvent}

Selection of appropriate solvent is an essential requisite for any reaction. The right solvent was selected by examining the activity in different solvents of varying polarity. The results are tabulated in Table 6. From the table, it can be deduced that reaction proceeds with great vigour in aqueous medium. Other solvents like isopropanol, acetone and dioxan permit the reaction to occur only in a nominal rate. However, high hydroquinone selectivity is observed over isopropanol and dioxan.
Table 6

Effect of solvent

\begin{tabular}{llll}
\hline Solvent & Conversion $(\%)$ & \multicolumn{2}{l}{ Seleccivity $(\%)$} \\
\cline { 3 - 4 } & & Catechol & Hydroquinone, \\
\hline Water & 77.2 & 64.1 & 3.9 \\
lsopropanol & 3.4 & 36.7 & 63.3 \\
Acctone & 5.5 & 69.0 & 31.0 \\
Dioxan & 4.9 & 36.3 & 63.7 \\
\hline
\end{tabular}

Phenol:solvent: $\mathrm{H}_{2} \mathrm{O}_{2}$ (molar ratio), 1:5:5; catalyst, $0.1 \mathrm{~g}$; temperalure, $80: \mathrm{C}$; time. $45 \mathrm{~min}$.

The high activity in aqueous medium can be recognised, with strong adsorption of phenol on the catalyst in this sol-' vent, which is driven by non-ideality of water phenol system. At the same mole fraction, the calculated activity coefficient of phenol is much higher in water than in other solvents [28]. When using organic solvents, their oxidation can be a competitive reaction system. However, products other than catechol and hydroquinone were not detected in the product mixture indicat-' ing that the oxidation of the solvent is not occurring in presence, of phenol.

\subsection{Effect of solvent concentration}

Oxidation of phenol is also a means for removing the organic pollutant from water streams. Thus, the amount of water in the system becomes critical. Hence influcnce of water concentration' was studied by varying the amount of water used, keeping the amount of phenol, oxidant and catalyst constant. The observa-" tions are outlined in Fig. 7. From the figure it can be inferred that at low concentrations, the amount of solvent plays a critical role. Phenol conversion increases with increase in the amount of water and after an optimum phenol to water molar ratio of 1:5, it remains constant.

The influence of water concentration has been studied" using organic peroxides in hydrocarbon oxidation [29]. It was concluded that active water species interact with peroxy radicals. The propagation and termination steps are influenced by water via hydrogen bond interaction of peroxy radicals. The same is applicable in oxidation of phenol also. The interaction of active water species with peroxy radicals increases with

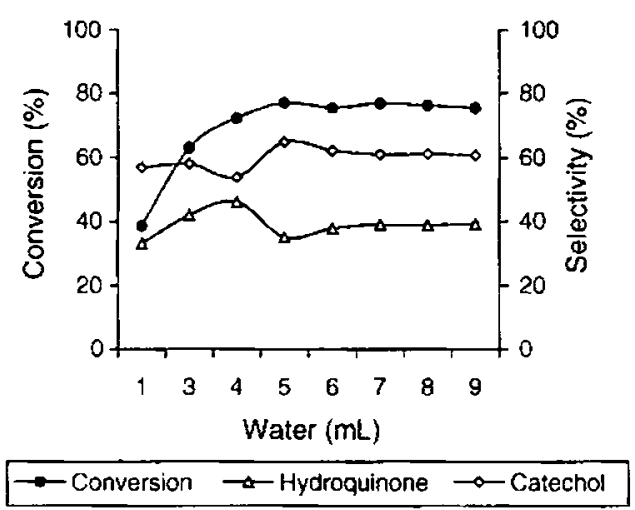

Fig. 7. Effect of solvent concentration. Phenol: $\mathrm{H}_{2} \mathrm{O}_{2}$ (molar ratio), 1:5: catalyst,

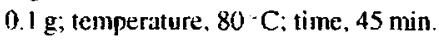




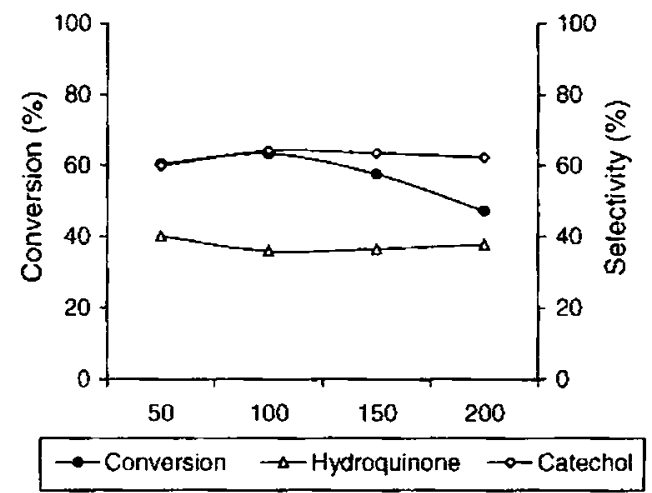

Fig. 8. Effect of catalyst concentration. Phenol:solvent: $\mathrm{H}_{2} \mathrm{O}_{2}$ (molat ratio), 1:5:5; temperature, $80^{\circ} \mathrm{C}$; time, $45 \mathrm{~min}$.

water concentration and hence the increased conversions at high amounts of solvent.

\subsection{Effect of catalyst concentration}

The catalyst amount required for maximum conversion of phenol was found out in the specified reaction conditions. The observations of the study are sketched in Fig. 8. From the figure, it can be noted that phenol oxidation increases with catalyst concentration. However, after an optimum amount of catalyst, the activity falls. Catechol selectivity also increases with increase in catalyst and then levels off.

From the figure, it can be concluded that a minimum amount of catalyst sites is required for the easy occurrence of the reaction. Increase in catalyst concentration accelerates decomposition of hydrogen peroxide [30]. The increased amounts of active sites in concurrence with increase in catalyst concentration can also lead to readsorption of products to active sites. Santos et al. reported a vital contribution of homogencous reaction at low catalyst loadings [31]. They suggested a homogeneous reaction for oxidation of phenol, while oxidation of intermediates is mainly due to heterogeneous mechanism. Yu et al. reported that excess of catalyst has no benefit on phenol hydroxylation and large excess of calalyst reduced the yield significantly [25].

\section{Mechanism of the reaction}

Several mechanisms have been proposed for the hydroxylation of phenol. Since the oxidant is hydrogen peroxide, most of them are based on free radicals, though elecrophilic mechanism has been postulated over titano silicates [4]. Meyer el al. suggested a helerogencous-homogenous free radical mechanism for liquid phase oxidations over solid acid catalysts [32]. This mechanism was applied for phenol successfully by Sadana and Katzer [33] and Lui et al. [34]. In this mechanism, free radicals are formed on the surface of solid acids, which subsequently undergo propagation and termination in solution. Two ways have been suggested for the gencration of free radicals on catalyst surface [35]. In the first mechanism, catalyst accelerates the decomposition of hydrogen peroxide into radicals. This ini-

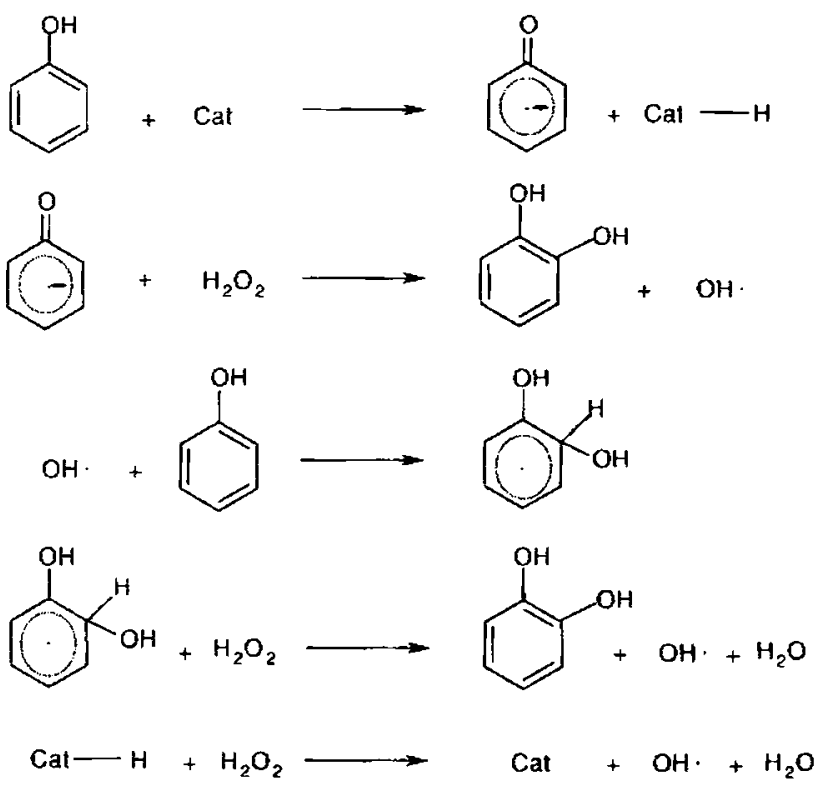

Fig. 9. Plausible mechanism for phenol hydroxylation with hydrogen peroxide.

tiation step is followed by the propagation step in solution. In the second mechanism, catalyst activates the phenol molecules directly and facilitates formation of phenoxy radicals, bringing about initiation. Franco et al. proposed that thermodynamically $o$ - and $p$-substitutions are equally feasible; kinetically substitution at $o$-position is more favoured. This suggests a steric effect on reaction, which can be explained in terms of a transition stage involving a complex with the $\mathrm{OH}$ of phenol and $\mathrm{OH}$ radical of the peroxide [9].

In the present study, an initial induction period was noticed in all cases, after which the reaction occurred violently with bursting of reaction mixture. Also, slight increase in temperature resulted in several fold enhancement in catalytic activity. Hence a free radical mechanism can be envisaged for the reaction. From solvent effect studies, it was concluded that adsorption of phenol on calalyst surface was favoured in aqueous medium. This suggests that formation of phenoxy radical in the catalyst surface is the rate determining step. Also, homogeneous contribution was not observed from catalyst concentration studics. The suggested mechanism involves formation of phenoxy radical on catalyst surface, which then reacts with peroxide giving $\mathrm{OH}$ free radical and diphenol. The produced free radical propagates the chain by attacking phenol molecule, forming diphenols. Same mechanism is proposed for the formation of isomeric products. The kinetic preference for catechol is explained by selective formation of hydroquinone inside the porous network of pillared clay. The proposed mechanism is sketched in Fig. 9.

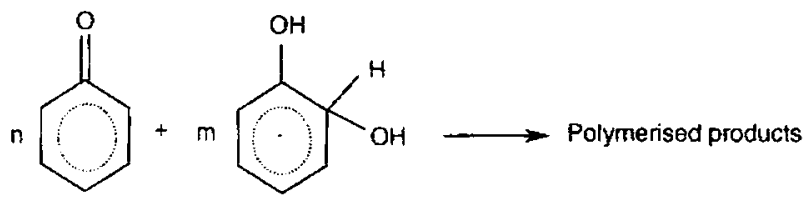

Fig. 10. Plausible side reaction during phenol hydroxylation. 
Intermediates formed in propagation step can lead to side reactions, forming polymerised products and this accounts for tar formation. This is depicted in Fig. 10.

\section{Conclusions}

Important outcomes that can be summarised from a systematic study of the reaction are:

- Mixed iron aluminium pillared clays and their transition metal exchanged analogues are good oxidation catalysts. The decrease in hydroquinone selectivity can be attributed to the shape selective nature of pillared clays.

- Correlation of catalytic activity with Brönsted acidity of the catalysts yields excellent results except for FeAl PM.

- Time has a promotional effect on the hydroxylation of phenol. An induction period of about $10-15 \mathrm{~min}$ is observed. Hydroquinone selectivity increases with time. Oxidation of phenol increases with temperature and maximum conversion is observed at $80^{\circ} \mathrm{C}$, after which, it drops. Hydroquinone selectivity decreases with tempcrature.

- Water was the best solvent for the reaction over pillared clays. Other solvents like acetone, dioxan and isopropanol show nominal conversion. Conversion increases linearly with solvent concentration. After an optimum value of solvent, the conversion\% levels off.

- Phenol conversion is almost independent of concentration of phenol. However, hydroquinone selectivity increases at higher amounts of phenol. After an initial increase, the conversion\% decreases with increase in catalyst concentration. Selectivity is independent of the amount of catalyst.

- Hydrogen peroxide concentration plays a promotional role on hydroxylation of phenol. After an optimum concentration of the oxidant, the activity levels off. Hydroquinone selectivity is independent of oxidant taken.

\section{Acknowledgement}

Manju Kurian thanks CSIR, New Delhi for Senior Research Fellowship.

\section{References}

[1] M.G. Clerici, in: M. Guisnet, J. Baebier (Eds.), Heterogeneous Catalysis and Fine Chemicals, vol. III.

[2] H. Jeifert. W. Wldmann, W. Sch Weidel. Ger. Patent 2.410 (1975) 742.
[3] P. Maggioni, US Patent 3,914 (1975) 323

[4] U. Wilkenhoner, G. Langhendries, F. van Laar, G.V. Baron, D.W. Gammon, P.A. Jacobs, E. van Steen, J. Catal. 203 (2001) 201-212.

[5] T. Yokoiu. P. Weng, T. Tatsumi, Catal. Commun. 4 (2003) II-15.

[6] F.S. Xiao. J. Sun. X. Meng. R. Yu, H. Yuan, J. Xu, T. Song, D. Jiang, R. Xu, J. Catal. 199 (2001) 273-281.

[7] C.W. Lee, D.W. Ahn, B. Wang, J.S. Hwang, S.-E. Park, Microporous Mesoporous Mater. 44-45 (2001) 587-592.

[8] A. Dubey, S. Kannan, S. Velu, K. Susuki, Appl. Catal. A 238 (2003) 319-326.

[9] L.N. Franco. I.H. Perez, A. Pliego, A.M. Franco, Catal. Today 75 (2002) $189-195$

[10] T.J. Pinnavia, Science 220 (1983).

[11] A. Gil, L.M. Gandia, M.A. Vicente, Catal. Rev. Sci. Eng. 42 (2000) $145-212$.

[12] K. Manju, S. Sugunan, Ind. J. Chem. 42A (2003) 2480

[13] K. Manju. S. Sugunan, React. Kinet. Catal. Lett. 81 (2004) 57.

[14] F. Bergaya, N. Hassoun. J. Barraoult, L. Gatineau, Clay Miner. 28 (1993) 109.

[15] D. Plee. F. Borg. L. Gatineau, J.J. Fripiat, J. Am. Chem. Soc. 107 (1985) $2362-2369$.

[16] D. Zhao, G. Wang, Y. Yang. X. Guo, Q. Wang, J. Ren, Clays. Clay Miner. 41 (1993) 317 .

[17] T. Sano, Y. Uno. Z.B. Zang, C.H. Ahn, K. Soga. Microporous Mesnporous Matcr. 31 (1999) 89-95.

[18] A. Tuel, S.M. Khouzami, Y.B. Taarit, C. Naccache, J. Mol. Catal. 68 (1991) 45-52.

[19] J.A. Martens, P. Buskens, P.A. Jacobs, A. vander Pol, J.H.C. vant Hoff, C. Ferrini, H.W. Kowenhoven, P.J. Koyman, H. Bekkum, Appl. Calal. A: Gen. 99 (1992) 71.

[20] H.L. del Castillo, A. Gil, P. Grange, Clays Clay Miner. 44 (5) (1996) 706-709.

[21] L.K. Boudali, A. Ghorbel, D. Tichit, B. Chiche, R. Dutarte, F. Figueras, Microporous Mater. 2 (1994) 525-535.

[22] D. Rohan. B.K. Hodnett, Appl. Catal. A: Gen. 151 (1997) 409

[23] B.J. Wittingtton, J.R. Anderson, J. Phys. Chem. 97 (1993) 1032.

[24] T. Blasco, J.M. Nicto, Appl. Catal. A: Gen. 127 (1995) 117-142.

[25] R. Yu, F.-S. Xiao, D. Wang, G. Pang, S. Feng, S. Qiu. R. Xu, Catal. Lett. 49 (1997) 49.

[26] S. Hamoudi, A. Sayari, K. Belkacemi. L. Bonneviot, F. Larachi, Catal. Today 62 (2000) 379-384.

[27] R. Neumann, M. Levin Elad, Appl, Catal, A: Gen. 122 (1995) 85.

[28] S.L. Saudler, Chemical and Engineering Thermodynamics, second ed., Elsevier, 1992

[29] L.J. Csanyi, K. Jakey, J. Catal. 141 (1993) 721-724

[30] J. Sun, X. Meng, Y. Shi, R. Wang, S. Feng. D. Jiang, R. Xu, F.-S Xiao, J. Catal. 193 (2000) 199-206.

[31] A. Santos, E. Barrasso, F.G. Ochoa, Catal. Today 48 (1999) $109-117$.

[32] C. Meyer, G. Clement, J.C. S Balaceanu, Procecdings of the 3rd Inter-t national Congress on Catalysis, vol. I, 1965.

[33] A. Sadana. J.R. Katzer, J. Catal, 35 (1974) 140.

[34] C.B. Lui, Z. Zhang, X.G. Yang, Y. Wu. J. Chem. Soc. Chent. Commun. $1019(1996)$

[35] C. Xiong, Q. Chen, W. Lu, H. Gao, W. Lu, Z. Gao, Catal. Lett. 69 ! (2000) $231-236$ 